\title{
Les Fables de La Fontaine ou l'assomption de la forme brève
}

Patrick Dandrey

Université de Paris-Sorbonne

M ots-clé: fable, La Fontaine, Esope, forme brève, poésie.

Keywords: fable, La Fontaine, Aesop, short narrative, poetry.

On ne mesure que rarement, hors de France, l'importance du nom et de l'œuvre de La Fontaine, et le rôle plus que littéraire, celui d'une adhésion et d'une identification culturelles, que ses Fables jouent depuis des siècles tout au long de la vie des Français, de la prime enfance à la vieillesse. L'on pourrait dire, sans exagération, que connaître les Fables de La Fontaine, c'est en savoir déjà beaucoup sur le pays qui les a vu naître et les cultive avec une attention si jalouse que, périodiquement, les Français se souvenant qu'Ésope a inspiré leur poète de référence se partagent entre ceux, amateurs de scandale, qui crient à la découverte d'une imposture surprenante, et ceux qui, farouches défenseurs du patrimoine national, ne seraient pas loin de soupçonner Ésope d'avoir par anticipation pillé La Fontaine. Plus sérieusement, on peut bien dire que la relation du fabuliste français avec son prédécesseur grec équilibre assez exactement la part de la filiation et de l'émancipation. Au titre de la fidélité à l'esprit ésopique, on peut rappeler que La Fontaine a restitué à l'apologue cette netteté de la forme binaire, cette forte articulation entre récit et moralité, cette structure à la fois dynamique et spéculaire qui dans l'apologue antique mettait en reflet et en dialogue le même propos distribué entre récit et moralité, chacune des deux parties traitant selon son génie propre le matériau ainsi réitéré. L'usage de la fable, jusqu'à l'abus, par les sermonnaires, les orateurs et les moralistes, avait tendu à brouiller sinon à dissoudre cette fermeté structurelle. Infidèle pourtant à son modèle, La Fontaine n'a pas craint de projeter en poésie ce qui demeurait essentiellement, intrinsèquement un genre prosaïque, à peine pittoresque, essentiellement moral. En cela plus proche peut-être de Phèdre, le fabuliste latin que pourtant il ne cite guère, préférant rapporter ordinairement son projet et ses sources à 
I'esclave de Phrygie: le premier recueil de 124 fables qu'il publie en 1668 est précédé d'une Vie d'Ésope Phrygien, sans ambiguïté. Mais le titre de Fables choisies et mises en vers par M. de La Fontaine souligne la transformation accomplie:le choix du vers, plus même qu'une question de forme, engage un parti esthétique, celui du déplacement en poésie d'un genre que sa nature originelle et son usage contemporain définissaient comme didactique: exercice de formation scolaire, instrument de probation et d'illustration rhétorique du discours éloquent, objet de méditation morale et lieu commun de sagesse partagée, l'apologue n'attendait rien moins que l'entrée sur le Parnasse.

Et en tout cas, pas en cohorte. Car l'on avait certes ici ou là tracé déjà en France le chemin que La Fontaine élargirait en boulevard. Mais les tentatives isolées de Marot, de Ronsard, ou, plus près de lui, celle de Mathurin Régnier tournant en satire une fable de «la Lionne, du Loup et du Mulet» ne représentaient pour eux que des exercices isolés, piquantes incursions sans lendemain d'un poète butinant une fleur de I'herbier ésopique. Seule Marie de France, au Moyen Âge, avait tourné en vers une centaine de fables ésopiques, renouvelant l'entreprise de Phèdre envers Ésope. Mais la comparaison de ces pièces à l'élégance un peu sèche, un peu roide, d'inspiration morale appuyée, met en nette évidence la différence avec la fantaisie capricieuse, variée, ornée, avec la subtilité des teintes réunies dans l'arc-en-ciel de pensée et de sensibilité qui haussent les Fables du seul La Fontaine au rang de poèmes, comme nous entendons cela aujourd'hui la chose: la versification se contentant tout au plus de faire indice, indice de quelque chose qui la dépasse, indice de cette modification de registre et d'intention esthétiques par quoi écriture narrative et écriture poétique excèdent de bien loin la distinction purement formelle entre prose et vers. Bref, vêtir de la «livrée des Muses», avec tous ses ornements de haute couture et ses ambitions de haute culture, les acteurs familiers des petits contes ésopiques - passait encore si c'était une fois et isolément; mais en faire une habitude jusqu'au système, quelle hardiesse! C'était prendre l'exception pour règle.

D'autant que dix ans après la première livraison, une petite centaine de nouvelles fables (87 exactement) devaient s'additionner aux précédentes dans un second recueil (1678-1679), et le tout s'enrichir encore en 1693, au soir de la vie du fabuliste, de plus d'une trentaine de poèmes, certains à peine encore identifiables à des fables, et publiés d'ailleurs auparavant parmi les Contes. En tout cas, se trouvait ainsi constitué un ensemble articulé en douze livres, comme une Énéide en miniature. Le premier vers de la dédicace de l'ouvrage au Dauphin de France, fils de Louis XIV, ne pastichait-il pas le premier vers du poème virgilien: «le chante les héros dont Ésope est le père/ Arma virumque cano»plaisante ouverture pour cette Iliade ou cette Énéide de poche qui s'amusait à doubler sa référence ésopique d'une allusion épique? Enfin, rappelons-le, cette audace tranquille et cette liberté conquise qu'autorisa peu à peu à La Fontaine sa longue fréquentation du genre, quelque trente ans durant, s'étaient doublées au fil du temps d'un renouvellement de ses sources, le filon oriental des fables persanes et indiennes, découvertes et introduites 
en France sous le nom de Pilpay, lui ayant suggéré l'addition, entre 1668 et 1678, de nouveaux apologues inspirés par ce modèle exotique, après l'épuisement (relatif) du fonds et de l'inspiration ésopiques dont la première livraison avait tourné le meilleur.

S'en était suivie une évolution de la forme même du genre, la stricte relation entre récit et moralité et les données de leur répartition, de leur expansion et de leur tonalité réciproquement définies s'en trouvant bouleversées après avoir été, au début de l'entreprise, restituées dans leur état originel, comme on vient de le dire. Créateur d'un genre quasiment neuf par provignement à partir de la souche ésopique, La Fontaine aura ainsi exploité les libertés que confère la poésie jusqu'à modeler ses fables sur des idylles, des églogues, des comédies ou des élégies, en y incluant de longues péroraisons en forme d'«applications», en fédérant, comme c'est le génie aussi des «Classiques» français ses contemporains, diverses influences, diverses tonalités, divers arts même: s'inspirant de l'emblème, genre iconographique, de la comédie, genre dramatique, du prône, genre rhétorique, l'apologue en tire une manière toute nouvelle quoique toujours dans le sillage de son modèle premier. Ainsi firent les La Bruyère, auteur des Caractères, les La Rochefoucauld, auteur des Maximes, les Charles Perrault, auteur des Contes, eux aussi héritiers d'un ou de plusieurs prototypes anciens ou antiques dont ils dégagent leur originalité, eux aussi jalonnant leur vie d'une pratique continue ou du moins notable du genre qu'ils façonnent à leur manière, eux aussi composant à partir de fragments une totalité unie et pourtant diverse, un collier offert à l'enfilement de perles nouvelles au fil des rééditions de leurs ouvrages en état de perpétuelle refonte. C'est la fécondité des genres brefs, qui autorise l'évolution du modèle par variation sans qu'en soit trahie l'essence, et qui par le biais du recueil permet l'enrichissement de la collection sans attenter à la concision des pièces collectionnées.

Car à la croisée des diverses révolutions esthétiques que nous venons d'évoquer, la brièveté fait office de pivot et d'enjeu. II n'est pour s'en convaincre que de revenir à la première de toutes, à cette révolution hardie qui consista à métamorphoser en poésie un genre prosaïque, didactique, démonstratif et narratif. Le génie de la forme brève, ses contraintes et ses contradictions, jouent leur plein rôle en cette affaire. Pour s'en convaincre, il n'est que d'entrer dans le détail de cette décision esthétique si originale, en commençant par en rétablir I'historique. Ce à quoi I'on va s'attacher maintenant.

Sans que I'on sache exactement comment La Fontaine vint à la rencontre de la fable, du moins peut-on par l'examen de ses débuts dans les Lettres déduire quelques hypothèses sur ce cheminement. On ne manquera pas d'y noter le rôle, déjà, qu'y jouait un penchant pour la brièveté que le genre de l'apologue lui permettra de satisfaire, même si ce fut, on le verra, au prix de distorsions et de contradictions au demeurant fructueuses.

Le jeune avocat qui dans le milieu de la décennie 1640 est venu de sa Champagne natale achever son droit à Paris, y a rencontré une compagnie de jeunes littérateurs qui 
se disent «Chevaliers de la Table ronde» et seront un jour les gens de Lettres en vue dans la capitale du Roi Soleil à son zénith. Pas des plus célèbres pour nous, mais non des moindres en leur temps: ils se nomment Pellisson, Furetière, Tallemant, Chapelain, Conrart, Charpentier. Certains plus âgés, comme le parlementaire Olivier Patru, ferré en éloquence gallicane, leur servent de mentors. C'est l'époque où La Fontaine, cultivant l'inspiration moyenne et élégante, choisit de tourner une comédie d'après Térence, broche un conte spirituellement libertin d'après les vieux diseurs français et italiens de la Renaissance, prépare surtout une idylle héroïque, cet Adonis imité de Virgile et d'Ovide qui constitue une sorte d'épopée en miniature, de taille plus brève et de registre plus modéré que l'épique et ses ambitions de sublimité. Nous avons déjà usé ici même de ce terme de miniature: prenons-y garde, il va constituer un leitmotiv de la carrière de notre poète, il suggère un penchant au modèle réduit, un goût pour la transposition délicate et enjouée des grandes formes à une échelle inférieure, que ce soit d'une réduction en longueur ou en «hauteur», comme on peut le dire en un temps où la hiérarchie des formes se mesure en degrés d'élévation, de I'humble au sublime, mais au moment précis où leur évaluation, par un génie de la proportion que la France hérite alors de l'ancienne Grèce, prétendra de plus en plus ignorer cette échelle, chaque niveau d'inspiration, de forme et de style pouvant prétendre à l'excellence dans son ordre. Le tout serait, dès lors, de proportionner exactement le traitement de l'œuvre d'art à son sujet et au genre qu'on lui assignait.

Le fabuliste allait merveilleusement jouer de ces dispositions esthétiques, en situant le traitement qu'il assigne au genre de l'apologue en vers entre l'humilité naturelle de son modèle (l'apologue ésopique est au bas de l'échelle hiérarchique des mérites et des ambitions) et la hauteur des genres sur lesquels il le modèle, certes à distance de pastiche, d'allusion, de complicité cultivée, mais sans craindre d'insérer ici ou là, au fil du traitement qu'il leur applique, des traits relevant des registres héroïque ou tragique, de l'inspiration philosophique ou morale, de la réflexion politique ou de la verve satirique. Le décalage subtil ou criant entre les deux niveaux situe le poème dans un entre-deux plaisant, exact équivalent esthétique de la dualité essentielle du genre, qui vouait un conte bouffon, fantaisiste, absurde, où les animaux parlent comme des hommes et les hommes se conduisent souvent en bêtes, à délivrer une morale à fin instructive ou méditative. La justesse de ton, la mesure à ne pas excéder, l'élégance discrète se combineront ainsi avec la brièveté originelle qui caractérise le genre, ou pour mieux dire ils en découleront, pour produire un objet de culture délicat et ciselé, un bijou dont la taille, si elle avait voulu excéder la miniature, aurait paru monstrueuse: forme brève, l'apologue l'était par nature. À vouloir se gonfler, comme la Grenouille qui se veut faire aussi grosse que le Bœuf, elle aurait explosé. Ainsi comprise, la brièveté naturelle de sa forme ne constitue plus seulement une question de structure ou une convention de principe, elle contribue à un équilibre fragile, effet d'une élaboration 
virtuose et méditée. C'est le génie de la miniature à la française, exalté par les enluminures médiévales, par les peintures de Fouquet ou les gravures de Callot.

Cette physique raffinée, La Fontaine l'a rencontrée et se l'est appropriée à la faveur d'une autre rencontre capitale dans sa formation et son aventure spirituelle: en 1657, il a été présenté au fastueux surintendant des finances Nicolas Fouquet. Celui en qui chacun voit alors, bien à tort, le futur premier ministre du jeune roi Louis XIV, le successeur de Mazarin lorsque commencera le règne personnel du monarque, est un amateur d'art, un adepte et un modèle de ce goût élégant et délicat, plus mesuré, plus souriant que I'héroïsme du règne précédent, plus distancé, moins appuyé que le burlesque du temps de la Fronde, mélange d'atticisme assoupli et de badinage mondain. Fouquet, ce Gulbenkian à la française, avait commencé à ériger lui aussi, dans les années 1650, sa fondation: le domaine de Vaux, près de Melun, où œuvrent pour lui les Le Brun, Le Nôtre, Le Vau, et dont La Fontaine sera chargé en 1658 de décrire les merveilles en cours d'édification: il le fera sous la forme à la fois cultivée et capricieuse, diverse et délicate du «songe». La mode est alors à la résurrection des genres oubliés, au mélange délicat des tons et des registres, que l'on nomme «tempérament», à l'enjouement et à la surprise. Le projet de composer des Fables en vers parées des grâces enjouées et badines de cette esthétique raffinée procède assurément du goût de ce milieu élégant, qui privilégie les genres brefs, car rien n'offusque tant le goût mondain que ce qui languit et ennuie, qui affectionne surprise et virtuosité, car dans ces milieux de plaisirs l'on se lasse vite du trop connu, du trop facile, et qui n'aime rien de ce qui pèse ou qui pose, au point de railler la mode précieuse pourtant issue de son cadre. La forme brève de l'apologue, assortie, par jeu et pour jouer, d'ornements raffinés en subtil et plaisant décalage avec son modèle, voilà qui devait ravir l'attente de ce public.

C'est tout justement ce que La Fontaine se flatte d'avoir tenté, après avoir, écrit-il dans la préface de son premier recueil,

considéré que, ces fables étant sues de tout le monde, je ne ferais rien si je ne les rendais nouvelles par quelques traits qui en relevassent le goût. C'est ce qu'on demande aujourd'hui. On veut de la nouveauté et de la gaieté. Je n'appelle pas gaieté ce qui excite le rire; mais un certain charme, un air agréable qu'on peut donner à toutes sortes de sujets, même les plus sérieux. (Collinet, 1991: 6)

C'est ici pourtant que le bât pouvait blesser, la difficulté surgir. Quand il pose ce principe esthétique qui définit admirablement tout le projet des Fables, c'est au fil d'un raisonnement où le procédé prend tout au plus l'allure d'une compensation par rapport à un autre idéal plus conforme à l'esthétique du genre auquel son génie personnel peut-être, le goût du temps sans doute, et plus encore l'intuition propre qu'il possède de la poésie véritable ne le portaient pas. Cet idéal, c'est, nous dit-il, celui de la brièveté, justement. Mais d'une brièveté toute pure, toute nue: sinon draconienne, attique au moins, sans les grâces ioniennes que va s'employer à y ajouter le nouveau 
fabuliste. Enjeu si central apparemment de son entreprise, que la question lui avait servi quelques pages plus haut à ouvrir cette même préface:

L'indulgence que l'on a eue pour quelques-unes de mes fables me donne lieu d'espérer la même grâce pour ce Recueil. Ce n'est pas qu'un des Maîtres de notre Éloquence n'ait désapprouvé le dessein de les mettre en Vers. II a cru que leur principal ornement était de n'en avoir aucun: que d'ailleurs la contrainte de la Poésie, jointe à la sévérité de notre Langue, m'embarrasseraient en beaucoup d'endroits, et banniraient de la plupart de ces récits la brièveté, qu'on peut fort bien appeler l'âme du Conte, puisque sans elle il faut nécessairement qu'il languisse. (ibid.: 4)

Tout est dit: la circulation de quelques fables transformées en poèmes dans les salons et bureaux d'esprit du temps, qui les ont aimées, a conduit le poète à en tenter un recueil complet; mais un maître vigilant, dans lequel nous reconnaissons Olivier Patru, à qui son ancien compagnon de la Table ronde n'a pas manqué de soumettre son ouvrage, a tout de suite relevé la contradiction entre ce que la poésie suppose d'expansion et la concision inhérente au genre. Patru lui-même, grand exemple de la belle éloquence gallicane qui trace sa voie au beau style du Classicisme français, venait en 1659 de tourner en prose deux fables ésopiques, pour modèle, dans le cadre d'un recueil de lettres galantes, dites À Olinde (Patru, 1681: 750-757) ${ }^{1}$. Suivis d'une volée d'applications morales et politiques, ces deux récits étaient, de fait, composés dans la plus vigilante concision, imitant les langues anciennes moins prolixes que le français. L'invention propre de Patru se révélait, en revanche, dans le commentaire greffé sur la moralité, dont la fécondité honorait l'imagination du moderne héritier du modèle.

Tout autre est le projet de La Fontaine. Se défendant de l'interdit jeté sur le vers, il appelle à la rescousse l'exemple de Phèdre. Mais, se détachant immédiatement de ce modèle même, il avoue avec une fausse humilité son incapacité à atteindre «l'élégance ni l'extrême brièveté qui rendent Phèdre recommandable: ce sont qualités au-dessus de ma portée» (Collinet, 1991: 7). Qualités hors de sa portée, peut-être - mais ne seraientce pas plutôt des qualités hors de son intention? On verrait là volontiers un prétexte pour glisser vers une autre visée esthétique, une autre conception de la poésie, où l'élégance tient moins à la rigueur d'une simplicité froide qu'à la grâce que confère l'ornementation délicate et variée:

Comme il m'était impossible de l'imiter en cela, j'ai cru qu'il fallait en récompense égayer l'ouvrage plus qu'il ne l'avait fait. Non que je le blâme d'en être demeuré dans ces termes: la langue latine n'en demandait pas davantage; et si l'on y veut prendre garde, on reconnaîtra dans cet Auteur le vrai caractère et le vrai génie de Térence. La

1 Lettres II, III et IV de la seconde partie. 
simplicité est magnifique chez ces grands hommes; moi qui n'ai pas les perfections du langage comme ils les ont eues, je ne la puis élever à un si haut point. II a donc fallu se récompenser d'ailleurs: c'est ce que j'ai fait avec d'autant plus de hardiesse, que Quintilien dit qu'on ne saurait trop égayer les Narrations (ibid.: 7)

La pente de l'auteur, le génie de la langue, l'autorité de Quintilien et le goût du moment auront ainsi conspiré à cette substitution esthétique : la convenance entre forme narrative et brièveté attique, définissant l'aptum esthétique de l'apologue selon Phèdre ou Patru, laissera place chez La Fontaine à la combinaison paradoxale de la brièveté narrative et de l'ornementation poétique. C'était le prix à payer pour le passage d'une définition formelle de la poésie (tourner en vers une narration brutale et sèche) à une définition matérielle (quand l'ornement, matière ajoutée au conte, transpose le narratif en poétique).

Est-ce à dire pourtant que cette transfiguration se paie au prix de la brièveté, et met en échec l'esprit même de la forme brève? Sans doute pas, comme un exemple va nous le montrer: nous parierons plutôt qu'elle en accomplit les exigences par d'autres voies. N'allons pas plus loin que les premiers vers de la toute première fable, la si célèbre Cigale et la Fourmi:

\author{
La Cigale, ayant chanté \\ Tout l'été,
}

Se trouva fort dépourvue

Quand la bise fut venue.

Pas un seul petit morceau

De mouche ou de vermisseau.

(v. 1-6)

Magnifique exemple de cette connivence difficile en apparence, merveilleusement efficace, en fait, entre la brièveté elliptique et de la périphrase alanguie associant et intervertissant leurs effets contrastés pour rendre le récit plus suggestif sans l'allonger outre-mesure, simplement en compensant les expansions par autant d'ellipses: un été entier tient en autant de syllabes qu'il a de mois («tout l'été» tient dans le mètre trisyllabique, le plus court des Fables); un début d'hiver se déploie, lui, dans une périphrase («quand la bise fut venue»), glosant en cinq mots et sept sons un instant à peine, celui où la bise survint - ou plutôt celui où elle est survenue: car elle est déjà là, sans qu'on l'ait sentie venir. Effet de surprise et de délectation microscopiques, comme cette évocation pittoresque des mets ordinaires de la Cigale, petit morceau de mouche ou de vermisseau: I'ornementation se fait familière, qui avait cédé au style élevé pour l'évocation du temps où «la bise fut venue». En six vers, La Fontaine a consommé le mariage contre-nature de l'ellipse et de la périphrase, du style élevé et familier. C'est du grand art, et si grand que rien là n'a paru durer, les accélérations compensant les ralentis. 
Mais si c'est du style heureusement poétique, il y manque encore pour que cela devienne pleinement ce que nous nommons poésie, cette mystérieuse consommation du sens dans l'expression qui définit l'essence poétique. Or, rien de moins difficile que de l'y déceler aussi. Car l'interversion plaisante et badine entre une si courte évocation de l'été et une si longue description du moment où le beau et le bon temps bascule dans les rigueurs de la disette ne constitue pas seulement un effet facile ou plaisant. C'est aussi une bien significative interversion, instructive et parlante, qui ne résorbe pas son charme dans une délectation purement formelle et spirituelle. Car comment mieux peindre le contraste entre la rapidité avec laquelle la Cigale a vu, ou plutôt n'a pas vu, passer l'été qu'elle a tout occupé à chanter, et la durée, l'interminable durée d'un moment, dès lors que la bise l'occupe de son souffle glacial, la faim de son appel insatiable et l'anxiété de sa morsure imparable? Un poète n'a pas besoin d'écrire un traité pour formuler la distinction entre le temps astronomique et la durée psychique: quatre vers y pourvoient, qui délivrent une leçon morale de prudence et esquissent une intuition philosophique sur les données immédiates de la conscience, à travers une suggestion verbale et rythmique.

Or, tout cela est si vite donné, si vite passé, que bien souvent le lecteur inattentif n'y prendrait pas garde. Pourquoi, sinon parce que rien de la brièveté du conte n'est sacrifié dans cette densité de l'évocation tantôt brève, tantôt longue, dont au finale tout paraît mesuré à la taille réduite de la forme brève et des insectes la peuplant? Poussons à la fin du petit récit. Une conversation en miniature esquissant les traits d'une comédie s'y termine par la chute bien connue: la Cigale, aux temps chauds, chantait au lieu d'épargner prudemment en vue des moments difficiles? Réplique de la Fourmi:

- Vous chantiez? J'en suis fort aise:

Eh bien! dansez, maintenant!

Ornementation, de nouveau: un mot piquant, presque un trait d'esprit, une nasarde pour faire sourire les salons. Mais pas seulement: car sous le mot, une allusion se glisse. De chanter à danser, la proximité littérale cache un décalage allusif. Chanter, c'est au sens propre, pour la cigale, crisser de ses élytres, exalter le soleil en insecte bruyant, cela évoque directement ses mœurs d'insecte. Mais lui suggérer de danser, c'est l'envoyer voir ailleurs, au sens conservé par l'expression française moderne «envoyer balader» (la balade vaut la danse); c'est aussi l'inviter à «danser devant le buffet», expression consacrée pour évoquer la famine. Or la Cigale n'est pas insecte danseur, la chorégraphie ne fait pas partie de ses mœurs. L'adresse convient plus aux hommes qu'aux bêtes. Bref, elle contient toute la morale de l'histoire: et c'est pourquoi cette fable ne déduit pas explicitement sa morale. Un verbe au sens ambivalent lui aura suffi. Veut-on meilleure brièveté? Sauf qu'il ne s'agit pas d'une brièveté simple et drue, mais finement ornée et habilement suggérée. 
Mieux: réduire la portée de sens de la fable à cette rude leçon de prudence assenée aux étourderies enfantines, ce serait méconnaître la signification humaine qui se dégage de tout le récit, de l'affrontement entre ses deux protagonistes, de leurs silhouettes, de leur dialogue; une signification plus riche, complexe, délicate que la prescription contenue dans les maximes du bon Ésope. II y a de la peinture morale derrière cette prescription moraliste, de l'analyse cachée dans cette leçon. Et de fait, même dans les très nombreuses fables où la moralité ésopique est conservée, la saveur archaïque de ces maximes, leur tour de prudente bonhomie, le sourire de connivence qui enveloppe leur délivrance, ne prétendent guère plus qu'à confirmer de vieilles certitudes, et plus souvent à amuser par le souvenir de ces vérités de toujours. La vraie philosophie des Fables, elle se découvre au fil du texte, de ses surprises, de ses aperçus soudains et rapides: un théâtre de silhouettes morales, de comportements stylisés, de conduites anatomisées transforme la vignette emblématique de l'original grec en une dramaturgie de l'âme et une comédie de la société qui en dit plus sur l'homme et sa destinée que toute la sagesse des nations véhiculée par les moralités ésopiques. Signe que le conte est entré en poésie, voici que le tour donné au récit délivre au revers de chaque phrase, de chaque image, une pensée, une vision du monde qui, le recueil s'étoffant vers toujours plus de profondeur discrète, de puissance suggestive et méditative, conduira au superbe poème philosophique qui conclut le XIIe livre: Ia fable du Juge arbitre, I'Hospitalier et le Solitaire, grande fresque méditative réduite aux dimensions d'un apologue. Rien là d'appuyé, en effet, La Fontaine connaît trop les règles de la brièveté justement mesurée: «Loin d'épuiser une matière,/On n'en doit prendre que la fleur» («Épilogue» du Premier Recueil). Fleur épanouie en bouquet - tel est le miracle de cette «brièveté ornée» qui colore les vignettes ésopiques sans les trahir, sans excéder leur concision, mais en accouchant le genre de ses virtualités de pensée, d'émotion et de coloration les plus secrètes.

Cette liberté rejaillit sur la structure esthétique de l'apologue: à force d'en varier la régulation, La Fontaine lui a conféré une souplesse qui, sans trahir son articulation majeure, son tour ni son ton, le rend capable de mimer en modèle réduit toutes ou presque toutes les formes de la littérature: la forme brève devient abréviation des formes longues, de bien des formes longues répertoriées par l'écriture de son temps. Ce génie de la miniature que révélait déjà le choix de l'idylle héroïque au temps d'Adonis, La Fontaine l'épanouit dans la fable renouvelée, transfigurée en miroir de sorcière, inscrit de manière spéculaire dans le tableau de la littérature dont il répercute sur le mode mineur le chorus varié, tant celui des formes que des thèmes, et celui aussi des sagesses et des écoles philosophiques, dont il essaie les diverses doctrines avec une désinvolture aimable, vaguement désillusionnée et pourtant toujours pertinente sous la forme abrégée qu'il en offre dans l'écrin minuscule de la fable. De la forme brève à l'esthétique de la brièveté ornée, son parcours aura conduit ainsi le fabuliste à proposer 
sous forme de recueil un abrégé d'art et de pensée qui collectionne dans les rayons de ses douze livres un compendium de la bibliothèque universelle. Peut-on rêver triomphe plus entier de la brièveté?

\section{Bibliographie}

\section{Ouvrages cités}

LA FONTAINE, J ean (1991). Euvres complètes. I-Fables, contes et nouvelles. Édition établie, présentée et annotée par J ean-pierre COLLINET. Paris: Gallimard, NRF, «Bibliothèque de la Pléiade».

PATRU, Olivier (1681). «Lettres à Olinde» (i. e. Mlle Le Vieux, 1659). [In] Plaidoyers et œuvres diverses de Monsieur Patru de I'Académie française. Nouvelle édition augmentée (1669). 2 parties en un vol. in- $8^{\circ}$. Paris: S.Mabre-Cramoisy.

\section{Ouvrages consultés}

Le Fablier. Revue des Amis de J ean de La Fontaine (BP 284, 02406 Château-Thierry), 1989.

BIARD, J ean-Dominique (1970). Le Style des Fables de La Fontaine. Paris: Nizet.

BORNECQUE, Pierre (1991). La Fontaine fabuliste. 4 éd. Paris: SEDES.

BRODY, J ules (1994). Lectures de la Fontaine. Charlottesville: Rockwood Pr.

BURY, Emmanuel (1996). L'Esthétique de La Fontaine. Paris: SEDES.

CLARAC, Pierre (1969). La Fontaine. Paris: Hatier.

(1958). éd. La Fontaine, J ean, Fuvres complètes. II-CEuvres diverses, édition établie et présentée par -Paris: Gallimard, NRF, «Bibliothèque de la Pléiade».

COLLINET, J ean-Pierre (1988). La Fontaine en amont et en aval. Pise: Goliardica.

(1992). La Fontaine et quelques autres. Genève: Droz.

(1970). Le Monde littéraire de La Fontaine. Paris: PUF. (rééd. Genève:Slatkine, 1989).

COUTON, Georges (1957). La Poétique de La Fontaine. Paris: PUF.

DANDREY, Patrick (1995). La Fontaine ou les métamorphoses d'Orphée. Paris: Gallimard.

(1992). La Fabrique des fables. Essai sur la poétique de La Fontaine. Paris, Klincksieck. (Rééd. abrégée: Poétique de La Fontaine. 1-La fabrique des fables. Paris: PUF, «Quadrige», 1996).

DUCHENE, Roger (1990). La Fontaine. Paris: Fayard.

FUMAROLI, Marc (1997). Le Poète et le roi. Jean de La Fontaine en son siècle. Paris: éd. de Fallois. éd. (1985). La Fontaine, J ean, Fables. Texte présenté et commenté par - . III. de M. Hugo. Paris: Imprimerie Nationale. (Livre de Poche, «Pochothèque», 1995). 
GUTWIRTH, Marcel (1987). Un merveilleux sans éclat: La Fontaine ou la poésie exilée. Genève: Droz.

JASINSKI, René (1966). La Fontaine et le premier recueil des Fables. Paris: Nizet.

KOHN, Renée (1962). Le Goût de La Fontaine. Paris: PUF.

MOURGUES, Odette de (1962). O Muse, fuyante proie... Essai sur la poésie de La Fontaine. Paris: J. Corti.

NEPOTE-DESMARRES, Fanny (1999). La Fontaine. Fables. Paris: PUF.

RUBIN, David Lee (1991). A Pact with Silence. Art and Thought in the Fables on Jean de La Fontaine. Ohio State U.P.

SPITZER, Leo (1928). Stilstudien. München: M. Hueber. (Études de style. Traduit de l'anglais et de I'allemand par Éliane Kaufholz, Alain Coulon, Michel Foucault. Paris: Gallimard, 1970, rééd. préfacée par J ean Starobinski, «Tel», 1993).

SWEETSER, Marie-Odile (1987). La Fontaine. Boston: Twayne Publ.

WADSWORTH, Philip A. (1952). Young La Fontaine. A Study of His Artistic Growth in His Early Poetry and First Fables. Evanston: Northwestern U.P.

Résumé: C réateur d'un genre quasiment neuf à partir du modèle ésopique, La Fontaine aura ainsi usé de toutes les libertés poétiques jusqu'à construire ses fables sur diverses influences, diverses tonalités et divers arts. La fable d'Esope souffre donc une évolution par variation, la brièveté faisant, chez La Fontaine, office de pivot et d'enjeu.

Abstract: By creating a virtually new genre based on Aesop's model, La Fontaine was keen to resort to all poetic innovations in order to build his fables in accordance with several influences, tones and arts. By adopting the Aesopian prescription of brevity, La Fontaine's fable therefore evolves through variation. 
